\title{
Quintana-Vallejo, Ricardo. (2021). Children of Globalization. Diasporic Coming-of-Age Novels in Germany, England, and the United States. Routledge.
}

\author{
Carolina UlloA \\ Posgrado en Letras ${ }^{1}$ \\ Universidad Nacional Autónoma de México | México \\ Contacto: cdanaeulloa@comunidad.unam.mx
}

1 Estudiante de la Maestría en Letras

U na labor crítica responsable es aquella que reconoce la humanidad detrás del objeto a tratar: aquella que enmarca, con sensibilidad, las materialidades que sostienen las temáticas de las representaciones culturales. En la compartida realidad contemporánea, que desde la teoría de los afectos se caracteriza por la acumulación de "both relatedness and interruptions of relatedness" (Gregg y Seigworth, 2010: 2 ), se vuelve imperante considerar los cuerpos detrás del artificio, "the challenge of making the writing equal to the subject being written about" (Probyn, 2010: 72). Como asevera Elsphen Probyn (2010), "writing affects bodies" (76), tanto de forma creativa como de forma académica. Children of Globalization. Diasporic Coming-ofAge Novels in Germany, England, and the United States (2021), del académico mexicano Ricardo Quintana-Vallejo, encarna este hecho: es un estudio crítico "about people whose bodies are displaced and whose minds are nurtured by globalized and artistic products" (142). Desde una trinchera distinta a aquella que lo vio nacer, este crítico investiga el género novelístico coming-of-age ${ }^{2}$ y los

2 Utilizo el término en el idioma original ya que, durante el recorrido teórico e histórico que Quintana-Vallejo (2021) provee, la traducción al español del género - "novela de formación" - no contiene los matices matices que adquiere en nuestra época, caracterizada por una globalización y multiculturalidad como nunca antes vistas.

Con tres partes que se dividen en ocho capítulos, la disertación inicia con la coincidencia generacional que llevó al autor a interesarse en el coming-of-age: la saga de Harry Potter de J. K. Rowling. Este vínculo personal y colectivo da pie a la discusión sobre el proceso de formación -las negociaciones con la identidad propia en el proceso de convertirse en adulto- de cualquier individuo, cómo el contexto define los parámetros del éxito de tal maduración y, en especial, cómo pueden (o no) adaptarse a ésta los grupos minoritarios. La investigación se ciñe a la diáspora; según Ricardo Ortíz, ésta se define como un desplazamiento geográfico que encuentra "identification with a group, however scattered, committed to the same work of cultural retention, reproduction, and revival of a home culture in an alien, foreign, 'host' setting' (cit. en Quintana-Vallejo, 2021:

que el autor propone en su estudio y definición del "coming-of-age" (4). Para él, éste es "an umbrella term that encompasses varied iterations of the genre [Bildungsroman] across history and the world [...] [that] share a common emphasis on the inner lives and internal drive of their protagonists, and necessarily showcase the formative process of a young person who becomes an adult" (4). 
144). Stuart Hall determina que los sujetos de la diáspora están "constantly producing and reproducing themselves anew, through transformation and difference" (cit. en Quintana-Vallejo 2021: 124) - y Quintana-Vallejo lo celebra y honra con un recorrido desde la tradición germánica del Bildungsromane y hasta el subgénero diasporic coming-of-age novels - En este libro atestiguamos, de la mano de un autor que ha experimentado en carne propia los encuentros y desencuentros que aborda, cómo se llevan a cabo las negociaciones identitarias entre la diáspora y los discursos nacionalistas monolíticos y opresivos que suelen dictar los estándares para su posible (des)identificación en el espacio que habitan.

La introducción sirve como marco teórico, pues se alimenta de los lineamientos tradicionales del Bildungsroman como los proponen Jerome Buckleyy Thomas Jeffers, clave para la asimilación y confrontación del arquetipo del héroe en el recorrido de Quintana-Vallejo (2021): "ablebodied protagonists from socioeconomically advantageous backgrounds [who] become idealized adults" (5). La primera parte, de carácter histórico, provee el panorama que inició la Bildungsroman y la reformulación en la tradición inglesa con obras tan aclamadas como David Copperfield y Great Expectations de Charles Dickens, autor que atraviesa este número de la presente revista. De esta manera, QuintanaVallejo desarrolla los fundamentos que permiten que las diasporic coming-of-age novels germinen en nuestros días y resalta su maleabilidad. Así, lo primordial para entender su objeto de estudio son las "internal forces of self-determination and the need to ponder on and experiment with one's identity" como "key drivers of the plot" (38), el puente que él ubica entre los protagonistas del siglo XIX y aquellos que emanan hoy.

Con un loable detenimiento crítico para interpretar los materiales literarios que sirven para su recorridohistórico, lasegunda partedel estudio, dedicada a tres grandes diásporas, ofrece una mirada a las "possibilit[ies] of non-belonging" (53) en las que se pactan los procesos de identificación y rechazo en ejemplos específicos. Con el estudio de Buddah of Suburbia, de Hanif Kureishi, y White Teeth, de Zadie Smith, comienza el cambio: los protagonistas ya no buscan una adultez idealizada, sino que tienen que cuestionarse cómo ellos "fit into national and global narratives" (59); en este caso, la convivencia de las comunidades de ascendencia india, bangladesí y jamaiquina en Londres. En el capítulo dedicado a la diáspora de México en Estados Unidos, Quintana-Vallejo hila su análisis a partir de los discursos de Donald Trump para explicar que las expectativas a las que los personajes de las diasporic coming-of-age novels se enfrentan están atravesadas por "contradictory and informed by racist, xenophobic, and mysoginistic imaginations of social hierarchies" (77). Estas dolorosas contradicciones se presentan mediante The House on Mango Street, de Sandra Cisneros, y Señales que precederán el fin del mundo, de Yuri Herrera, los cuales demuestran "the constant becoming of culturally-hybrid protagonists as they experiment and puzzle over their multiple identities" (99). Lo anterior enarbola "decolonial understandings of diasporic identities" (99) que llevan al crítico a explorar las Antillas Mayores, específicamente Puerto Rico y Cuba, y la potencia queer de los personajes de Down These Mean Streets, de Piri Thomas, y Antes que anochezca, de Reinaldo Arenas.

La tercera parte regresa al origen germánico tras las múltiples revaloraciones de lo que se entiende por "identidad nacional". Mediante Selam Berlin, de Yade Kara, y tschick, de Johann Gottfried von Herder, Quintana-Vallejo describe un panorama en el que las sociedades globales "become ever more comfortable with fluid national identities that can integrate difference without pushing for the cultural erasure that full assimilation implies" (132). Ambos textos niegan una conclusión organicista y, así, posibilitan que la audiencia confronte iteraciones monolíticas de lo que implica el crecimiento humano. Con esta premisa, el último capítulo diagnostica una 
posible vía en el futuro de este subgénero, uno en el que sus protagonistas demuestran una aguda consciencia de sus condiciones en la diáspora en comentarios de orden metatextual. ${ }^{3}$ Dichas preocupaciones subrayan la interseccionalidad de la construcción identitaria, pues, a la par de una opresión racial sistémica, los personajes $-\mathrm{y}$ las personas - se enfrentan a segregaciones de clase, orientación sexual y género (146). En las últimas obras analizadas, On Earth Were Briefly Gorgeous, de Ocean Vuong, y Juliet Takes a Breath, de Gabby Rivera, se cruzan los ejes que se habían delimitado gracias al estudio de los textos previos, como la diversidad sexual, el ejercicio escritural (storytelling) y, más que antes, las redes de apoyo entre las comunidades diaspóricas.

En los procesos de formación de los protagonistas de las diasporic coming-of-age novels se vierte el énfasis en la negociación: un sustantivo que, en sí mismo, ya habla de fluidez, una falta de estabilidad, que Children of Globalization celebra. Quintana-Vallejo no sólo ofrece un sugerente recorrido histórico y crítico para el subgénero, con riesgos interpretativos sanos de por medio, sino que plasma el inicio de una conversación crítica indispensable en nuestros tiempos: "Who am I and what is my place in this world?" (16). Bajo la premisa de Jeffers, quien explica que "the emergence of the heros character increasingly mirrored the emergence-socially, economically, politically, ideationally-of the world around him" (113), el crítico mexicano asevera que las novelas

3 Siguiendo la propuesta del narratólogo Gérard Genette. que analiza también son reflejos de su época. Por esta razón, los hijos a los que refiere el título del libro no sólo son los personajes protagonistas que el autor estudia, sino, también, somos nosotros. Hoy vale la pena mirar el drama de lo humano, en la compleja realidad que nos sostiene, y buscar herramientas críticas que nos ayuden a entenderlo de manera más cabal. Como concluye QuintanaVallejo, "The coming-of-age genre is more varied than ever and, more than ever, its readership is as complex as the characters it portrays" (160). Si bien ni las novelas ni la presente investigación dan respuestas a la pregunta que inicia este párrafo, Children of Globalization es una vía ideal para continuar reflexionando cómo "a body's never less than ongoing immersion in and among the world's obstinacies and rhythms, its refusals as much as its invitations" (Gregg y Seigworth, 2010: 1) en su forma más híbrida y multicultural.

\section{Referencias bibliográficas}

Gregg, Melissa; Seigworth, Gregory J. (2010). "An Inventory of Shimmers". En Melissa Gregg y Gregory J. Seigworth (Eds.), The Affect Theory Reader (pp. 1-25). Duke University Press.

Quintana-Vallejo, Ricardo. Children of Globalization. Diasporic Coming-of-Age Novels in Germany, England, and the United States. (2021). Routledge.

Probyn, Elspeth. (2010). "Writing Shame”. En Melissa Gregg y Gregory J. Seigworth (Eds.), The Affect Theory Reader (pp. 71-90). Duke University Press.

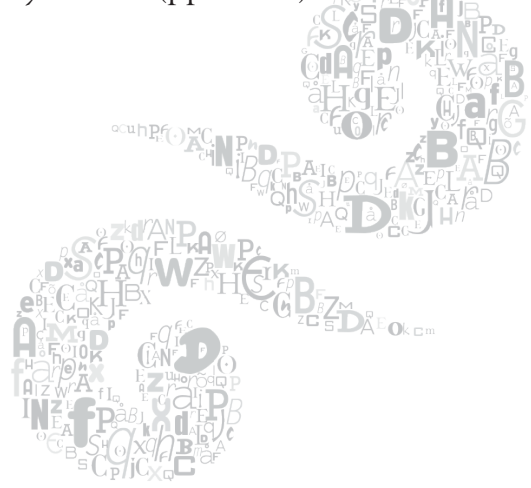

